\title{
Mitigation of the navigation signals from the pulsar observations based on the adaptive side-lobe cancellation
}

\author{
Da Huang ${ }^{1, a}$,Zhu Cheng ${ }^{2, b}$, Zhuang Wang $^{1, c}$ and Danmei Zhong ${ }^{2}$ \\ ${ }^{1}$ Science and Technology on Automatic Target Recognition Laboratory (ATR), Nation University of \\ Defense Technology, Changsha 410073, China; \\ ${ }^{2}$ College of Electronic and Engineering, Nation University of Defense Technology, Changsha \\ 410073, China. \\ ahuangda_nudt@sina.com, ${ }^{b}$ chengzhu88@gmail.com, 'czhuang_wang@sina.com
}

Keywords: the radio astronomy; anti-interference; the adaptive side-lobe cancellation; the navigation signal;

\begin{abstract}
Pulsar study is one of the important issues on radio astronomy; however, the quality of pulsar observations is affected by the spectrum overlaps with navigation signals. Actually, pulsar signals and navigation signals are usually overwhelmed in the noise. Thus this paper focuses on the application of observation data to build two evaluation indexes, which are the interference suppression ratio and the signal loss ratio. The method to eliminate navigation signals is guided by these two indexes. Combined with the spatial characteristic of navigation signals, this paper proposed a modified adaptive side-lobe cancellation method based on noise suppression. The noise component in the interference subspace is estimated and eliminated by the eigenvalue decomposition, and then the optimum weight vector is obtained. Finally navigation signals in observation data are effectively suppressed. Simulation results show that compared with the eigenspace-based algorithm for adaptive antenna side-lobe cancellation, the interference suppression ratio of the proposed method has been increased by 10 20dB.
\end{abstract}

\section{Introduction}

Pulsar plays an important role in the stellar evolution, the interstellar medium and other issues on the radio astronomy. L-band $(1 \mathrm{GHz} 2 \mathrm{GHz})$ is an important observation frequency band of pulsars, but this band is often used in many communication signals, such as satellite navigation signals, BEIDOU B3 (1268.52MHz), GPS L2 (1227.6MHz) etc. These communication signals have a certain bandwidth that overlaps with the observing spectrum of pulsar.

Although navigation signal spectrum overlaps the pulsar spectrum, navigation signals generally come from different directions. Therefore, the spatial characteristic of navigation signals can be used to eliminate its effect. The earliest usage of spatial characteristic is radar jamming. The adaptive sidelobe cancellation method is one of the effective and typical methods using the spatial feature.[1-3] . With the development of radio telescopes, new multi-channel radio antennas are gradually appearing. G.Hellbourg[4] , van der veen [5] and SJ Wijnhold [6] take advantage of multichannel spatial location, such as the interferometer(VLA) and the phase Phased array( LOFAR), to extract the interference subspace through eigenvalue-decomposition(EVD) of the covariance matrix. Then, the received signal is orthogonally projected on the interference subspace to eliminate interferences. However, the interference subspace is inaccurately estimated because the received signal includes the signal and the noise. Therefore, this signal is affected by non-orthogonality. G.Hellbourg[7] , BDjeffs[8] and van der veen[9] estimated a much accurate interference subspace by auxiliary antennas, and improved the performance of cancellation. The methods described above essentially use the orthogonality between the interference subspace and the noise subspace to obtain the optimal weight vector. Then the optimal weight vector makes the synthetic pattern to form nulls at the directions of interferences. The availability of these methods depend on whether the power of 
interference is greater than its noise. However, when the interference comes from the navigation signal, these methods are disabled because the power of the interference is weaker than the noise.

To solve these problems, firstly, this paper builds two evaluation indexes based on the application of the data in the radio astronomy. These indexes, which are interference suppression ratio and signal loss ratio, measure the degree of interference cancellation and signal loss, respectively. Also, they can provide a basic guidance on designing methods to eliminate navigation signals. Next, combined with spatial characteristics of navigation signals, this paper proposes a modified adaptive sidelobe cancellation method based on noise suppression. Then the optimum weight vector can be obtained by a better orthogonality between the subspace of the noise and the interference. Finally, based on the proposed indexes, this paper compares the proposed method and an eigenspace-based algorithm for adaptive antenna sidelobe cancellation (E-ASC) [3].

\section{Evaluation Indexes Construction}

\section{Basic questions statement}

In order to quantificationally analyze the impact of navigation signals in pulsar observations, we compare the power of each signal before and after the received system.

Because the received power of radio signals depends on the effective received area and the receiver bandwidth, it is necessary to distinguish intensity of each signals using the spectral power flux density. Jansky (abbreviation is $\mathrm{Jy}, 1 \mathrm{Jy}=10^{-26} \mathrm{~W} \cdot \mathrm{m}^{-2} \cdot \mathrm{Hz}^{-1}$ ) is a common used unit in the radio astronomy. In the pulsar table at $400 \mathrm{MHz}$, most pulsars concentrate below $5 \mathrm{mJy}$, which take up 49\%, while there are 7 stars of above $50 \mathrm{mJy}$, which take up 1.3\%. The radiation power of pulsars follows the law spectrum, so the higher frequency corresponds to the weaker flux.[10]

According to the ICD documents of GPS and BEIDOU, the minimum level of navigation signals is $-160 \sim-163 \mathrm{dBw}$ in the antenna with 3dbi $[11,12]$. This level can be converted to the spectral power

flux density which is $-196 \sim-201 \mathrm{dBw} /\left(\mathrm{m}^{2} / \mathrm{Hz}\right)$ in bandwidth (2.046 20.46MHZ). The pulsar of $1 \mathrm{mJy}$ is $-290 \mathrm{dBw} /\left(\mathrm{m}^{2} / \mathrm{Hz}\right)$ in the same bandwidth. Therefore, the power of navigation signals is higher 89 90dBw than the pulsar. Moreover, the receiver system can also affect the receiving signal power. It is necessary to consider the influences of the received system in the radio telescope (the main antenna) and the auxiliary antenna.

Generally speaking, the main-lobe of the main antenna points at the pulsar, and the interference (the navigation signal) comes from sidelobes. This paper takes the FAST30m[13] model pulsar receiver as the main antenna model to compare the power among the signal, the interference and the noise .The pattern of the FAST30m in L-band can be obtained by the GRASP[14] as shown Fig.1.b

The sky background temperature is usually $20 \mathrm{~K}$,but the low noise amplifier(LNA) will extra increase $80 \mathrm{~K}$. Therefore, the total noise temperature is about $100 \mathrm{~K}$. The receiver bandwidth of the FAST30m is about $100 \mathrm{MHz}$, and the effective lighting aperture is $18 \mathrm{~m}$. The receiving signals are amplified about 90dB by LNA, radio frequency amplifiers, intermediate frequency amplifiers, etc. Navigation signals have the fixed bandwidth which is less than the receiver bandwidth. As a consequence, the power of navigation signals is merely proportional to the effective received area. When the navigation signal of BEIDOU (bandwidth $20.46 \mathrm{MHz}$, center frequency B3 1268.52MHz) comes from the first sidelobe of FAST30m. Combined with Fig.1 and the spectral power flux density mentioned above, the calculated power of the interference is about $-20 \mathrm{dBm}$, the noise is $-60 \mathrm{dBm}$ and the pulsar of $1 \mathrm{mJy}$ is $-10 \mathrm{dBm}$.

The auxiliary antenna is multichannel for taking advantage of the spatial characteristic of navigation signals. Hence the auxiliary antenna consists of uniform linear array, which each array element has no directionality. If the element aperture is $20 \mathrm{~cm}$ and the receiver bandwidth is $22 \mathrm{MHz}$, combined with parameters mentioned above, we can conclude that the power of the pulsar and the 
navigation signal is $100 \mathrm{~dB}$ and $10 \mathrm{~dB}$ respectively, which is less than its noise. Thus the pulsar signal can be ignored.

From the analysis above, the power of the navigation signal, which comes from sidelobes of the main antenna, is less than noise in the pulsar observation. Therefore, this paper focuses on the problem how to suppress the power of navigation signal which is less than the noise power in pulsar observations.

\section{Evaluation indexes construction}

Cancellation ratio is a common index to show the ratio of the output signal power before and after the cancellation. It is more appropriate in the strong interference. When the interference power is much weaker than the noise power, the noise power is dominant in the received signal energy. Thus cancellation ratio cannot effectively show the variation degree of the interference and signal components. To solve this problem, this paper sets up two indexes, which are the interference suppression ratio and the signal loss ratio.

Generally speaking, the receiving signal of the main antenna before and after the cancellation can be respectively expressed as:

$$
\begin{aligned}
& y(t)=c_{S} F\left(\theta_{S}\right) S(t)+c_{I} F\left(\theta_{I}\right) I(t)+N_{p}(t) \\
& \tilde{y}(t)=c_{S} \tilde{F}\left(\theta_{S}\right) S(t)+c_{I} \tilde{F}\left(\theta_{I}\right) I(t)+\tilde{N}_{p}(t)
\end{aligned}
$$

Where $c_{S}, c_{I}$ are constants related to the power, $F\left(\theta_{S}\right), F\left(\theta_{I}\right)$ are values of the normalized directivity function of main antenna at $\theta_{S}$ (the signal)、 $\theta_{I}$ (the interference) directions, $S(t)$, $I(t)$ and $N_{p}(t)$ are the signal, the interference and the noise(White Gaussian Noise) in the main antenna, respectively. “ "denotes the variation after the cancellation. The receiving signal of the auxiliary antenna can be expressed as:

$$
x(t)=c_{I}^{\prime} F_{r}\left(\theta_{I}\right) I\left(t-t_{0}\right)+N_{r}(t)
$$

Where $c_{I}^{\prime}$ is a constant related to the power, $F_{r}\left(\theta_{I}\right)$ is the value of the normalized directivity function of the auxiliary antenna at $\theta_{I}$ (the interference) direction; $t_{0}$ is the time delay of the interference, $N_{r}(t)$ is the noise(White Gaussian Noise) in the auxiliary antenna. It should be noted that the signal, the interference, and the noise of both the main and the auxiliary antenna, are mutually unrelated. Then the interference suppression ratio can be defined as:

$$
G_{I}=\frac{\max \left[R_{x y}\left(\tau_{1}\right)\right]}{\max \left[R_{x \tilde{y}}\left(\tau_{2}\right)\right]}=\frac{\max \left\{E\left[y(t) \cdot x^{*}\left(t-\tau_{1}\right)\right]\right\}}{\max \left\{E\left[\tilde{y}(t) \cdot x^{*}\left(t-\tau_{2}\right)\right]\right\}}=\frac{\tilde{F}\left(\theta_{I}\right)}{F\left(\theta_{I}\right)} \frac{\max \left\{E\left[I(t) \cdot I^{*}\left(t+t_{0}-\tau_{1}\right)\right]\right\}}{\max \left\{E\left[I(t) \cdot I^{*}\left(t+t_{0}-\tau_{2}\right)\right]\right\}}=\frac{\tilde{F}\left(\theta_{I}\right)}{F\left(\theta_{I}\right)}
$$

Eq.(4) shows that the interference suppression ratio $G_{I}$ measures the correlation of the interference between the main antenna and the auxiliary antenna. The variation of the correlation reflects the variation of the pattern at the interference direction. Therefore, $G_{I}$ evaluates the variation of the interference component before and after the suppression.

Similarly, when $S(t)$ is a specific form, the signal loss ratio measures the correlation of the signal between the main antenna and the auxiliary antenna. Then the signal loss ratio can be defined as:

$$
G_{S}=\frac{\max \left[R_{S y}\left(\tau_{1}\right)\right]}{\max \left[R_{S \tilde{y}}\left(\tau_{2}\right)\right]}=\frac{\tilde{F}\left(\theta_{S}\right)}{F\left(\theta_{S}\right)}
$$

By Eq. (5), $G_{S}$ shows the variation of the pattern at the signal direction, and evaluates the variation of the signal component before and after the suppression. 


\section{Basic principle}

The system structure based on adaptive sidelobe cancellation is shown in Fig.1.a.

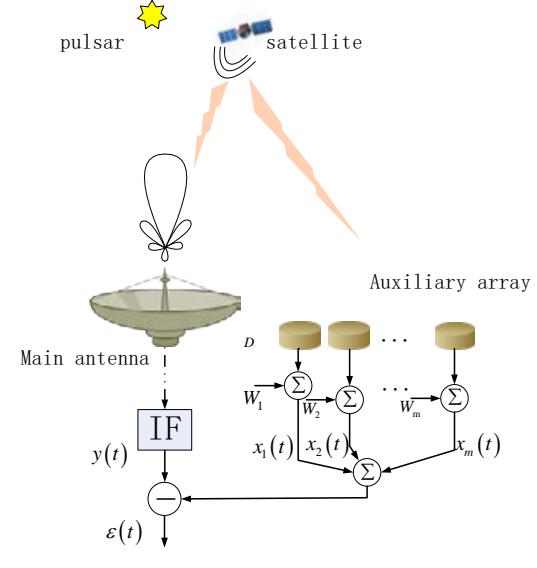

(a)

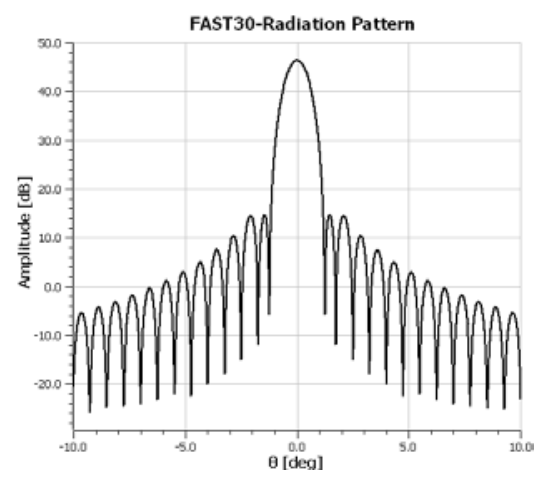

(b)

Fig.1 Schematic diagram of adaptive sidelobe cancellation, (a) and the pattern of the FAST30m, (b)

In Fig.1.a $y(t)$ is the received signal of the main antenna, $x(t)=\left[x_{1}(t), x_{2}(t), \ldots, x_{k}(t)\right]^{T}$ and $W=\left[w_{1}, w_{2}, \ldots, w_{k}\right]$ are the received signal and the weight vector of the auxiliary array, where $x_{i}(t)$ and $w_{i}$ are the received signal and the weight value of the $i$ th array element. $\varepsilon(t)$ is the difference between the signal of the main antenna and the weighted signal of the auxiliary array. The core issue of the adaptive side-lobe cancellation estimates the weight vector $W$.

The optimal weight vector $W_{\text {opt }}$ is obtained based on the orthogonality between the subspace of the interference and the noise in the E-ASC. The interference subspace can be extracted by the EVD of the covariance matrix of the auxiliary array, because the received signal of the auxiliary array mostly includes the interference and the noise. The covariance matrix can be estimated by the following formula:

$$
\hat{R}_{x}=\frac{1}{N} \sum_{i=1}^{N} x^{*}\left(t_{i}\right) x^{T}\left(t_{i}\right)
$$

Where $N$ is the number of snapshots. Assume there are $p(p<k)$ unrelated narrowband interferences, the EVD of the covariance matrix is shown as:

$$
\hat{R}_{x}=\hat{U}_{I} \hat{D}_{I} \hat{U}_{I}^{H}+\hat{U}_{N} \hat{D}_{N} \hat{U}_{N}^{H}
$$

Denote $\hat{D}_{I}$ and $\hat{D}_{N}$ as the diagonal matrix constituted by the former $p$ big eigenvalues and the later $k-p$ small eigenvalues. $\hat{U}_{I}$ and $\hat{U}_{N}$ are the matrixes made up of eigenvectors. The interference subspace and the noise subspace are formed by the column vectors of $\hat{U}_{I}$ and $\hat{U}_{N}$. When the interference is much greater than the noise, these two spaces are approximately perpendicular to each other. In order to obtain the optimal weight vector, the cross-correlation vector of the main antenna and the auxiliary antenna is:

$$
\hat{R}_{x y}=\frac{1}{N} \sum_{i=1}^{N} x^{*}\left(t_{i}\right) y\left(t_{i}\right)
$$

On account of limited snapshots, the weight vector is projected on the interference subspace to avoid the estimated error [3], as below:

$$
\hat{w}_{\text {opt }}=\hat{U}_{I} \hat{D}_{I}^{-1} \hat{U}_{I}^{H} \hat{R}_{x y}
$$

As discussed above, the power of the navigation signal is much weaker than the noise power in pulsar observations, so the eigenvalues of the interference and noise is quite close. The interference 
subspace is strongly perturbed by the noise. Therefore, the estimated error of the optimal weight by the formula (9) is very large.

The eigenvalues corresponding the noise subspace are almost the same because the white noise is stationary. Therefore, the noise component of the interference subspace can be estimated and eliminated through these eigenvalues. The specific steps are as follows:

(a) the covariance matrix and the cross-correlation vector can be estimated by Eq.(6)and(8);

(b) take the EVD of $\hat{R}_{x}$ as Eq.(7) and calculate the mean $\bar{\lambda}=\frac{1}{k-p} \sum_{i=p+1}^{k} \lambda_{i}$ by $k-p$ small eigenvalues;

(c) $\hat{R}_{x}$ minus the diagonal matrix with $\bar{\lambda}$, as follows:

$$
\tilde{R}_{x}=\hat{R}_{x}-\left(\frac{1}{k-p} \sum_{i=p+1}^{k} \lambda_{i}\right) I
$$

(d) Obtain the optimal weight vector by Eq.(9)

\section{Analysis of Simulation Experiment}

To further validate the effectiveness of the proposed method, this paper compares the proposed method and the eigenspace-based algorithm for adaptive antenna side-lobe cancellation (E-ASC), by using the proposed indexes.

\section{Simulation parameters setting}

This paper takes the FAST30m as the main antenna, which parameters are described in [13]. the uniform linear array of the auxiliary antenna is space between $0.1 \mathrm{~m}$ and the noise temperature is $200 \mathrm{~K}$. The pulsar signals of $1 \mathrm{mJy}$ are replaced by the random signal, because different pulsars have different forms and periods. The interference is the navigation signal of BEIDOU in B3 (1268.52MHz) and C/A code.

This paper quantitationally analyzes the performance of the proposed method and the E-ASC method in the aspects of the direction of interference arrival (DOA) (Fig.3), the observation time (Fig.4), the auxiliary arrays aperture (Fig.5), and the number of elements (Fig.6). Specific parameters settings is shown in Table 1.In addition, the Monte Carlo simulation method is applied to eliminate the evaluation random error. According to the law of large numbers, the Monte Carlo error is 0.0158 (the confidence level $\alpha=0.03$, the standard deviation is 0.0743 (the auxiliary antenna noise variance/ W ) and the experimental times of Monte Carlo is 200). Therefore, the results are sufficiently reliable.

Table 1 simulation parameters settings

\begin{tabular}{ccccc}
\hline Parameters & Aperture $/ \mathrm{m}$ & $\mathrm{DOA}(\theta))^{\circ}$ & Observation time/s & Elements number \\
\hline Fig2 & 0.5 & $0 \sim 5$ & 0.01 & 10 \\
Fig3 & 0.5 & 1.5 & $1 \mathrm{e}-5 \sim 5 \mathrm{e}-3$ & 10 \\
Fig4 & $0.1 \sim 5$ & 1.5 & 0.01 & 10 \\
Fig5 & 0.5 & 1.5 & 0.01 & $3 \sim 15$ \\
\hline
\end{tabular}

\section{Method performance analysis}

- DOA

There is a big difference of the interference power at different directions for the pattern. Thus DOA is discussed, as shown in Fig.2. 

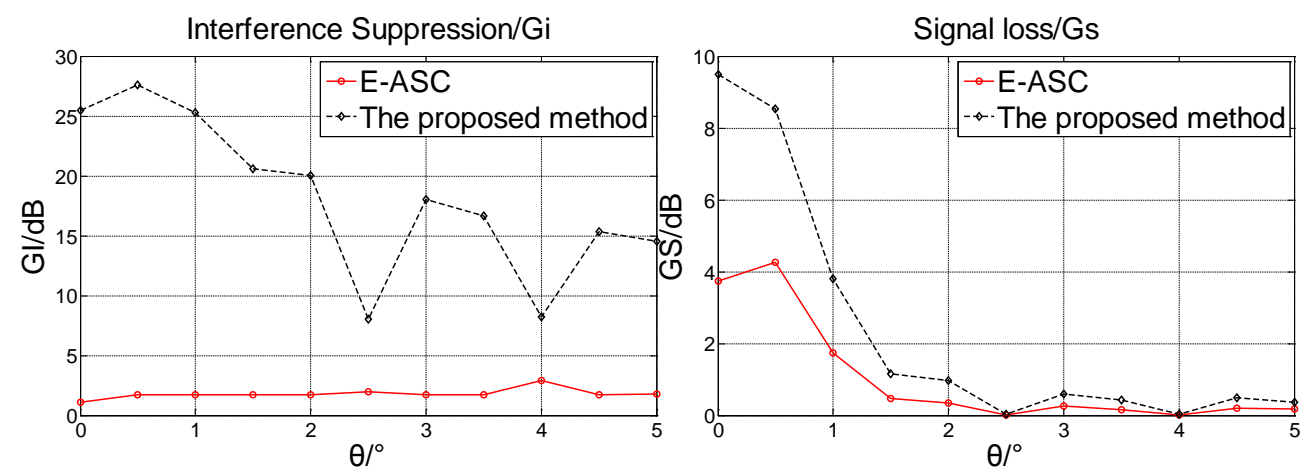

Fig.2 Comparison of different DOA

Combined with Table 1 and Fig.2, it is easy to draw the conclusion that $G_{I}$ of the proposed method decreases with DOA increasing and is overall 7 20dB higher than E-ASC; in the right picture of Fig.2, $G_{S}$ of the main lobe in $0.5^{\circ}$ is larger than the sidelobes, because nulls in the main lobe affect the signal. The deeper null will cause much loss of the signal. Consequently, $G_{S}$ of the proposed method is about $5 \mathrm{db}$ larger than E-ASC method. When the interference is far away from the main lobe, $G_{S}$ gradually tends to zero.

- Observation time

Observation time is corresponding to the number of snapshots $\mathrm{N}$ in the formula (6) and (8). Observation time will affect the estimation accuracy of the covariance matrix and the correlation vector, so observation time is discussed, as shown in Fig.3.
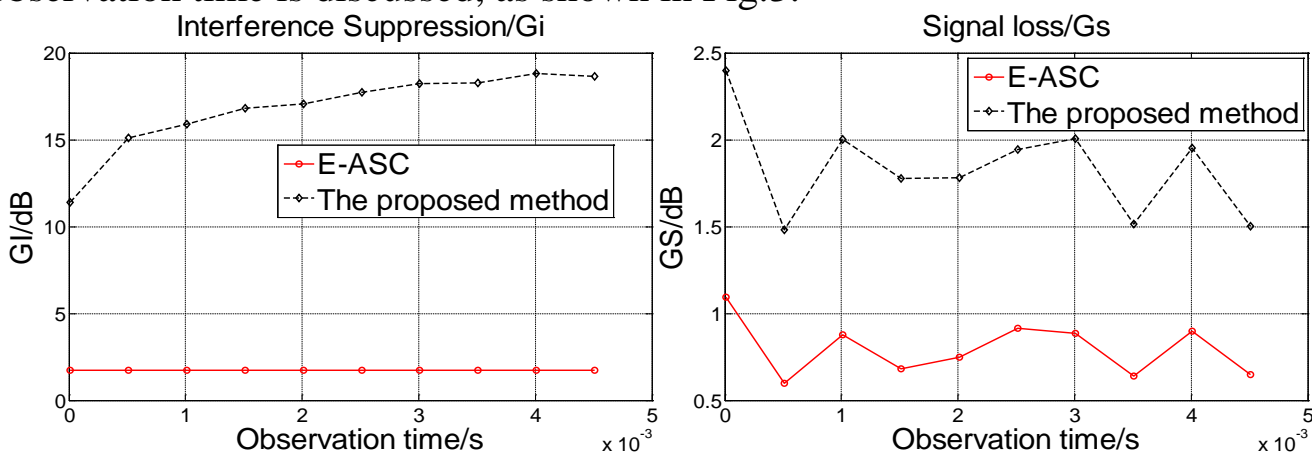

Fig.3 Comparison of different observation time

Combined with Table 1 and Fig.3, it is easy to conclude that the interference suppression ratio $G_{I}$ of the proposed method increases with the observation time, and is at least $10 \sim 18 \mathrm{~dB}$ higher than the E-ASC. Then the signal loss ratio $G_{S}$ is about $1 \mathrm{~dB}$ higher than the E-ASC.

\section{- Element aperture}

The array element aperture affects the power of the navigation signal, so the element aperture is discussed, as shown in Fig.4
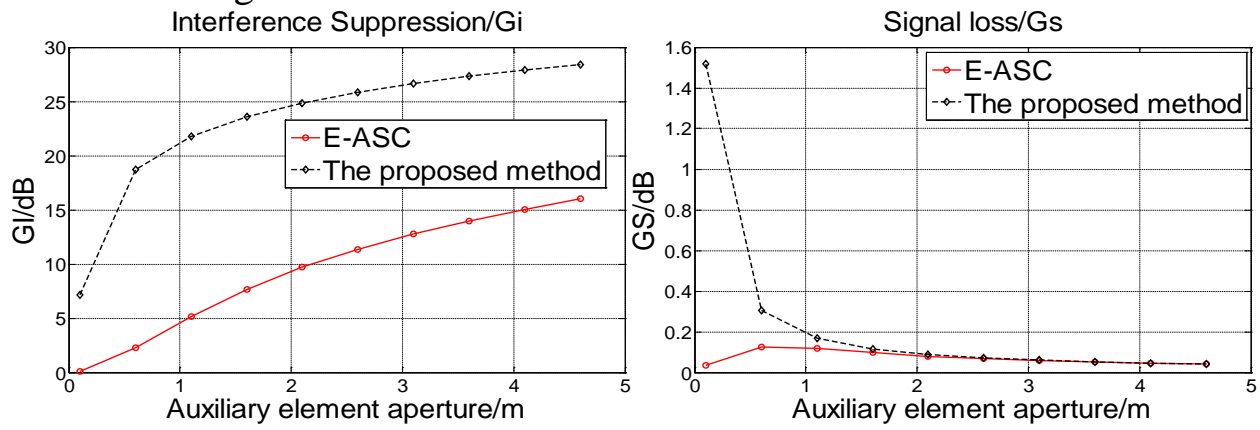

Fig.4 Comparison of different element aperture 
Combined with Table 1 and Fig.5, $G_{I}$ of both the proposed method and the E-ASC increase with the aperture, but $G_{I}$ of the proposed method is overall about $7 \sim 10 \mathrm{~dB}$ higher than the E-ASC. Meanwhile, the signal loss ratio $G_{S}$ tends to 0 with the increasing aperture.

- The number of array elements

The number of array elements affects the depth of nulls and the available number of nulls, so it is discussed, as shown in Fig.5.
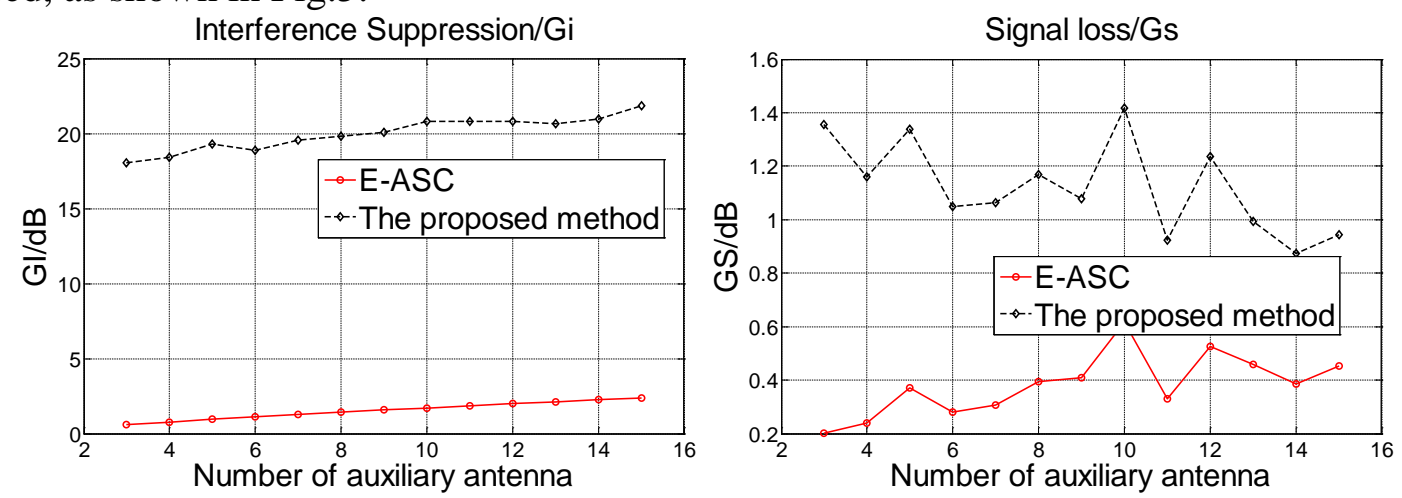

Fig.5 Comparison of the different number of auxiliary elements

Combined with Table 1 and Fig.5, the conclusion is that $G_{I}$ of both the proposed method and the E-ASC increase with the number of array elements. Moreover $G_{I}$ of the proposed method is overall about $15 \sim 20 \mathrm{~dB}$ higher than the E-ASC. At the same time, the signal loss ratio $G_{S}$ of the proposed method is approximately $1 \mathrm{~dB}$ higher than the E-ASC.

\section{Summary}

In the pulsar observations, the navigation signal, as the radio interference, is usually down the noise power, which leads to the invalidation of common indexes and the failure of traditional spatial methods. To cope with the two problems, first of all, this paper puts forward two indexes, the interference suppression ratio and the signal loss ratio, to reflect the extent of the interference suppression and the signal loss. Secondly, combined with the spatial characteristic of the navigation signal, this paper proposes a modified adaptive sidelobe cancellation method based on noise suppression, which the noise component in the interference subspace is estimated and eliminated through the eigenvalues of the covariance matrix. The proposed method makes the interference subspace and the noise subspace to have a better orthogonality, and then obtains the optimal weight vector. Compared with the E-ASC, $G_{I}$ of navigation signal in the proposed method increased by 10 $20 \mathrm{~dB}$, and the impact $G_{S}$ on pulsar signals is just higher 1 2dB than before.

\section{ACKNOWLEDGMENT}

This work is supported partly by the National Natural Science Foundation of China under Grant 11173068

\section{References}

[1] C.Yang-Ho, "Improved adaptiveing of coherent interference without spatial smoothing," vol. 52pp 3464-3469, 2004.

[2] C.Yi and H.Wei-Yau, "A Robust Algorithm for Adaptive Interference Cancellation," vol. 56, pp 2121-2124, 2008. 
[3] Z.Yongbo,W.Zhihui, and Z.Shouhong,"An eigenspace-based algorithm for adaptive antenna sidelobe cancellation," in Signal Processing. Proceedings. ICSP 2004 7th International Conference on, vol.3, pp 2086-2089, 2004.

[4] G.Hellbourg, T.Trainini, R.Weber, E.Moreau, C.Capdessus, and A.J. Boonstrd, "RFI subspace estimation techniques for new generation radio telescopes," in Signal Processing Conference (EUSIPCO) Proceedings of the 20th European, pp 200-20, 2012.

[5] A.J.van der Veen, A.Leshem, and A.J.Boonstra, "Signal processing for radio astronomical arrays," in Sensor Array and Multichannel Signal Processing Workshop Proceedings, pp 1-10, 2004.

[6] S. J.Wijnholds, A.J. van der Veen, F.De Stefani, E.La Rosa, and A. Farina, "Signal processing challenges for radio astronomical arrays," in Acoustics, Speech and Signal Processing (ICASSP) IEEE International Conference on, pp 5382-5386, 2014.

[7] G. Hellbourg, A. P. Chippendale, M. J. Kesteven, and B. D. Jeffs, "Reference antenna-based subspace tracking for RFI mitigation in radio astronomy," in Signal and Information Processing (GlobalSIP) IEEE Global Conference on,pp 1286-1290, 2014.

[8] B.D.Jeffs, L.Li, and K.F.Warnick, "Auxiliary antenna-assisted interference mitigation for radio astronomy arrays," vol. 53, pp 439-451, 2005.

[9] A.J.van der Veen and A.J.Boonstra, "Spatial filtering of RF interference in radio astronomy using a reference antenna," in Acoustics, Speech, and Signal Processing. Proceedings. (ICASSP '04). IEEE International Conference on, vol.2, pp 189-92, 2004.

[10]A.G.LYNE and R.T.RITCHINGS, "The binary pulsar observed at four radio frequencies," vol. 268, pp 606-607, 1977.

[11] "Navstar GPS Control Segment to User Support Community interfaces," in ICD-GPS-870A.15, 2011.

[12]C.S.N.Office, "BeiDou Navigation Satellite System Signal In Space Interface Control Document”, 2011.

[13] G. Jianjian, C.Yang, G.Henqian, and J.Chengjin, "Design,Manufacture and Test of L-band Feed for the FAST 30m Demonstrator," Astronomical Research \& Techinology, vol. 4,pp 48-52, 2007.

[14]http://www.ticra.com/products/software/grasp 